\title{
Pediatric Primary Malignant Lymphoma of the Spine: A Case Report
}

\author{
Daisuke Kudo*, Naohisa Miyakoshi, Michio Hongo, Yuji Kasukawa, Yoshinori Ishikawa, \\ Hiroshi Aonuma, Yoichi Shimada
}

Department of Orthopedic Surgery, Akita University Graduate School of Medicine, Akita, Japan.

Email: *dkudo@doc.med.akita-u.ac.jp

Received July $24^{\text {th }}, 2012$; revised August $26^{\text {th }}, 2012$; accepted September $14^{\text {th }}, 2012$

\begin{abstract}
Background: Cases of primary malignant lymphomas of the bone are rare and account for about $<1 \%$ of all lymphomas and 5\% of extranodal Non-Hodgkin's lymphomas. Furthermore, most reports have described the occurrence of this disease in the middle-aged population, pediatric malignant lymphomas originating in the bone, particularly in the spine is rare. Methods: A 10-year-old boy presented with low back pain caused by T12 vertebral compression fracture due to sustaining a fall. A month later, he still presented with prolonged low back pain that intensified after exercise. A neoplastic lesion in T12 vertebral body was identified after spine computed tomography (CT) and magnetic resonance imaging. Results: We performed CT-guided biopsy, and he was diagnosed with primary malignant lymphoma of the vertebral body. He was treated with multiagent chemotherapy without irradiation, and complete remission was maintained at the 5-year follow-up. Moreover, the height of the deformed vertebral body improved as he grew. Conclusions: Herein, we report a rare case of pediatric primary malignant lymphoma of the spine with successful clinical and radiological outcome.
\end{abstract}

Keywords: Malignant Lymphoma; Pediatrics; CT Guided Biopsy; Wrap around Sign; Chemotherapy; Irradiation

\section{Introduction}

Lymphomas are a heterogeneous group of malignancies affecting the $\mathrm{B}$ or the $\mathrm{T}$ cells; they usually originate in the lymph nodes but may originate in any organ of the body $[1,2]$. However, cases of primary malignant lymphomas originating in the bone are rare, and account for $<1 \%$ of all lymphomas [3] and $5 \%$ of extranodal NonHodgkin's lymphomas (NHLs) [4]. Most reports have described the occurrence of this disease in the middleaged population [5-7]. However, cases of pediatric malignant lymphomas originating in the bone, particularly in the spine, are rare, with an incidence rate of $<5 \%$ [810]. Herein, we report a rare case of pediatric primary malignant lymphoma of the spine in a patient showing a good clinical course and radiographic improvement.

\section{Case Presentation}

A 10-year-old boy presented with low back pain after sustaining a fall. Radiography performed at the first visit revealed a T12 vertebral compression fracture. He had no neurological abnormality and received only symptomatic therapy. A month later, he still presented with prolonged

${ }^{*}$ Corresponding author. low back pain that intensified after exercise. Reexamination of the radiographs of the spine revealed a wedge deformity of the T12 vertebral body (Figure 1). In addition, a neoplastic lesion at T12 was identified after computed tomography (CT) and magnetic resonance imaging (MRI). CT revealed a soft mass shadow around the T12 vertebral body, also known as a "wrap-around" sign, without destruction of the bone cortex and periosteal reaction (Figure 2). No other lesions were detected on whole-body CT. Sagittal T1-weighted MRI revealed an isointense lesion corresponding with a bulge located posterior to the vertebral body (Figure 3(a)), and T2weighted images revealed an isointense to hyperintense lesion. Axial MRI revealed a mass around the area surrounding the vertebral body and the spinal canal (Figure 3(b)). Contrast enhanced T1-weighted MRI revealed enhancement of the mass [11]. Technetium-99 m bone scintigraphy and Gallium-67 scintigraphy revealed only weak uptake in the area to the right of the T12 vertebra and showed no accumulation in other areas. Complete blood cell count revealed a reduced white blood cell count of $1500 / \mathrm{mm}^{3}$ (neutrophil count, $225 / \mathrm{mm}^{3}$; lymphocyte count, $1185 / \mathrm{mm}^{3}$ ), and no other abnormalities were detected in the biochemical blood analysis. Needle biopsy was performed under $\mathrm{CT}$ guidance through a pos- 


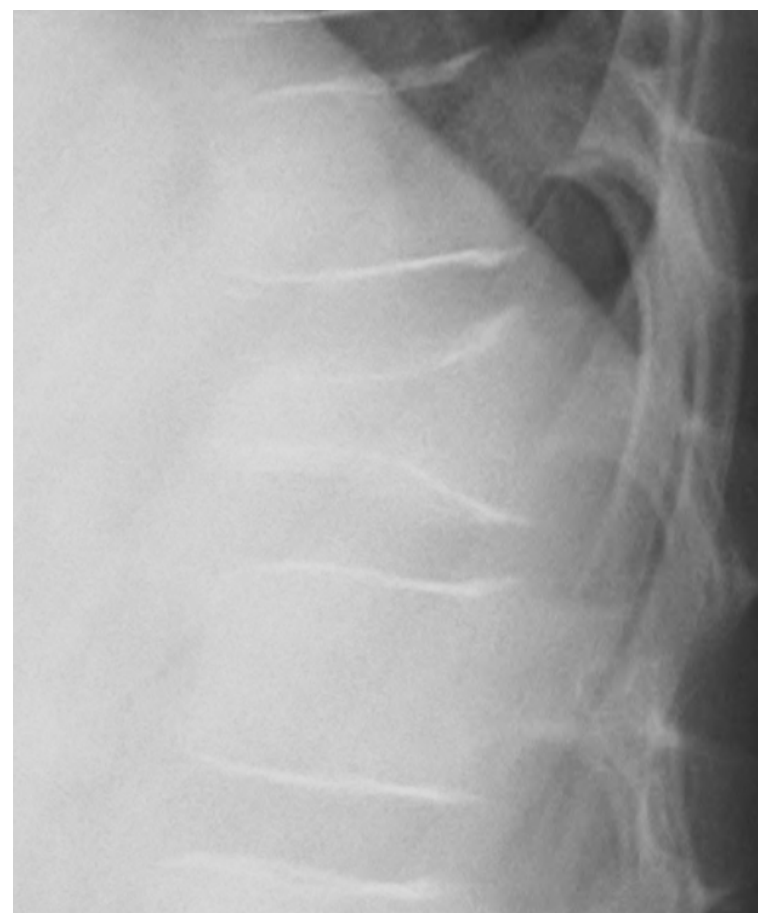

Figure 1. Radiography of the thoracic spine shows wedge deformity of $\mathrm{T} 12$ vertebral body.

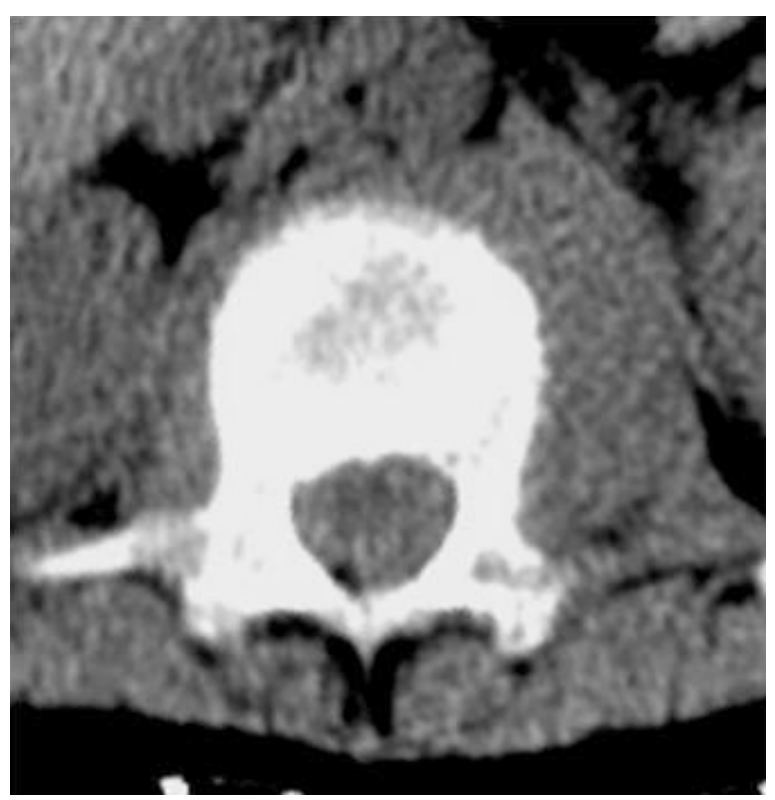

Figure 2. CT revealed a soft mass shadow around the vertebral body of T12, so-called "wrap-around" sign, without destruction of the bone cortex and periosteal reaction. This sign is a characteristic finding in malignant lymphoma of the spine.

terior approach. Histopathological examination revealed findings consistent with those of large B-cell lymphoma. Cerebrospinal fluid cytodiagnosis could not confirm the presence of tumor cells. On the basis of the above findings, he was diagnosed with primary NHL of the verte-

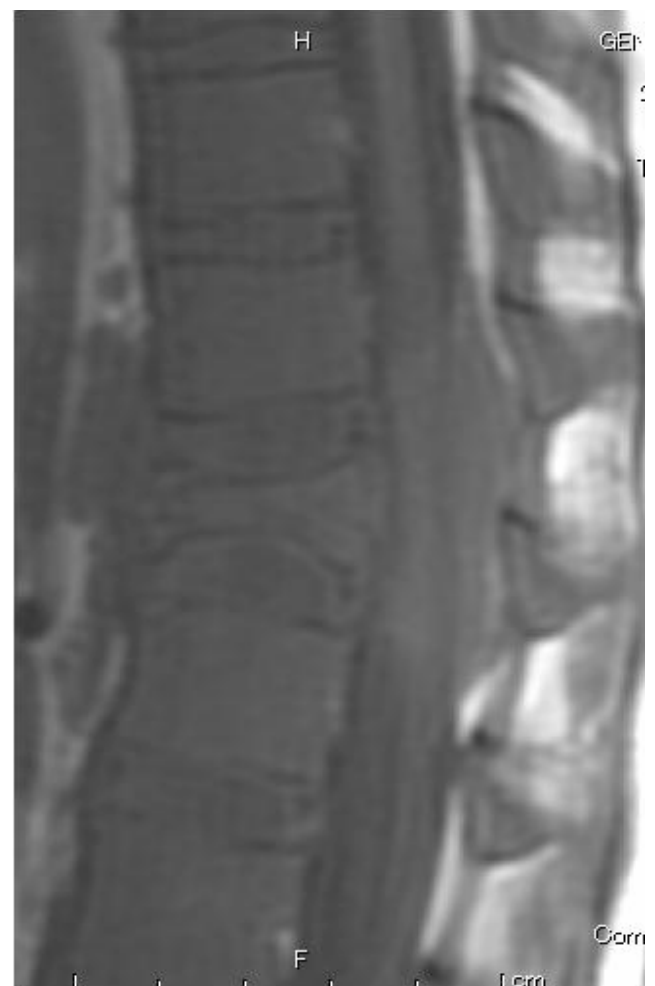

(a)

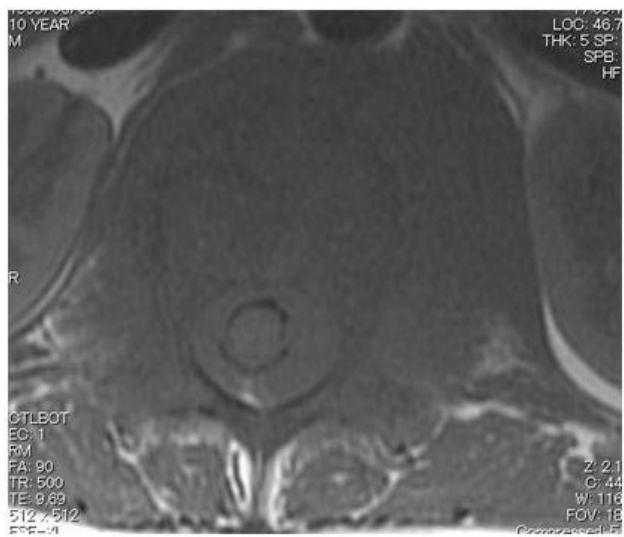

(b)

Figure 3. (a) Sagittal T1-weighted MRI revealed an isointense lesion corresponding with a bulge located posterior to the vertebral body; (b) Axial MRI revealed a mass around the area surrounding the vertebral body and the spinal canal, while the continuity of the bone cortex was maintained.

bral body as stage IV [12]. According to Phase II clinical trials of mature B-cell neoplasm in children: A Japanese Cooperative Studies, we performed short duration, intensive chemotherapy. In this regimen, all registered patients are stratified into 4 risk groups (G1, stage I/II and resected tumors; G2, stage I/II and unresected tumors; G3, stage III/stage IV central nervous system (CNS) negative; G4, stage IV CNS positive and Burkitt leukemia). Patients in G4 receive 2 courses of $\mathrm{A} 1, \mathrm{~A} 2$, and $\mathrm{B}$. Course A consists of dexamethasone, methotrexate, cyc- 
lophosphamide, vincristine, and pirarubicin; course B consists of cytarabine, methotrexate, vincristine, dexamethasone, and etoposide. Cranial irradiation is omitted in this protocol. Because the Lymphome Malins de Burkitt (LMB) 89 study reported that despite not receiving cranial irradiation but receiving an additional course of high-dose methotrexate $\left(8 \mathrm{~g} / \mathrm{m}^{2}\right)$ and intrathecal chemotherapy, children with CNS involvement had a similar outcome to those with CNS involvement treated with cranial irradiation, with 5-year event-free survival (EFS) of $79 \%$ [13]. Thus, we performed multiagent chemotherapy according to the G4 protocol. Unfortunately, 5 months after the initiation of chemotherapy, he sustained a fall again. Radiographs showed L1 and L2 compression fractures associated with steroid-induced osteoporosis with a Cobb angle of $2^{\circ}$ right sided coronal curvature and kyphotic angle of $33^{\circ}$ measured from T10 - L2 (Figure 5(a)). The deformity index (the ratio of vertebral body height to its longitudinal diameter) of T12, L1 and L2 were $1.00,1.54$ and 1.31 , and the kyphosis rate (the percentage of vertebral body height at the anterior region to its height at the posterior region) of T12, L1 and L2 were $25.2 \%, 70.7 \%$ and $59.1 \%$, respectively [14]. MRI scans showed fresh fracture of L1 and L2 without neoplastic lesion. His spine was immobilized using a hard corset. Seven months after the initiation of chemotherapy, the patient entered complete remission. MRI revealed no signs of tumor (Figure 4). Five years after disease onset, clinical complete remission has been maintained. In addition, spinal deformity improved as he grew. Kyphotic angle improved to $11^{\circ}$, but Cobb angle slightly worsened to $4^{\circ}$. The deformity index of T12, L1 and L2 improved to $1.97,1.76$ and 2.02 , and the kyphosis rate of T12, L1 and L2 improved to $92.1 \%, 78.1 \%$ and $86.7 \%$, respectively (Figure 5(b)).

\section{Discussion}

In 1901, Wieland first reported a case of primary malignant lymphoma of the bone [15]. Primary malignant lymphoma of the spine is also rare, accounting for $0.1 \%$ to $3.3 \%$ of all malignant lymphomas [10]. However, most reports have described the occurrence of this disease in the middle-aged population [5-7], and only few large case series of pediatric primary malignant lymphomas of the bone have been reported. Glotzbecker et al. have reported that a review of their institution's Pediatric Tumor Registry identified 15 cases of primary malignant lymphoma of bone from among 306 cases of NHL diagnosed between 1970 and 2003. They also reported that in 122 patients, only 24 of the 239 primary lesions with malignant lymphoma of the bone were located in the spine, as reported in similar case series [8]. Malignant lymphoma of the spine commonly has an initial presentation oflumbar backache with paraplegia or sphincter dysfunction. In

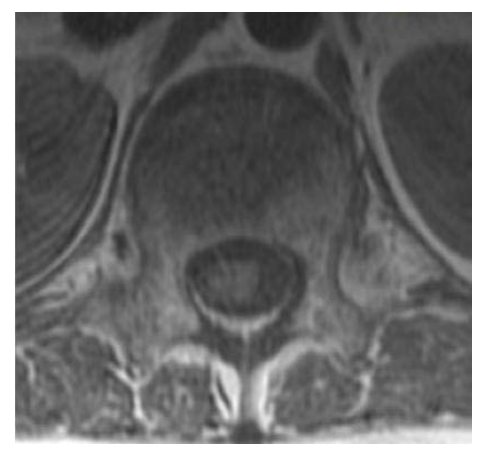

Figure 4. The tumor responded well to the chemotherapy. Seven months after the initiation of chemotherapy, MRI revealed no signs of tumor.

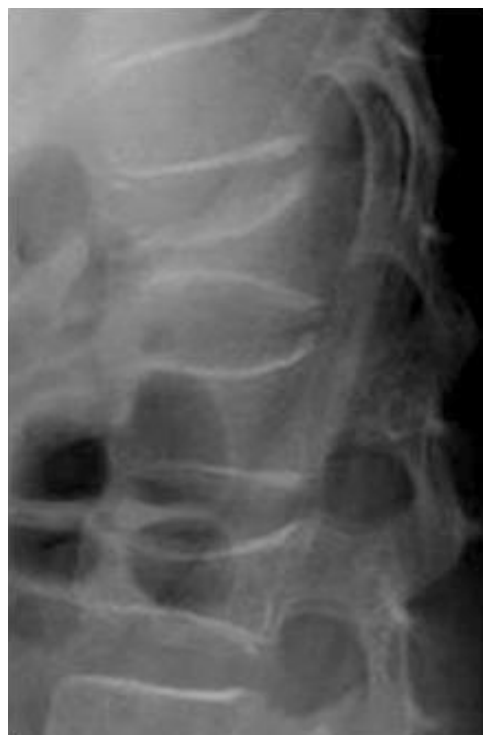

(a)

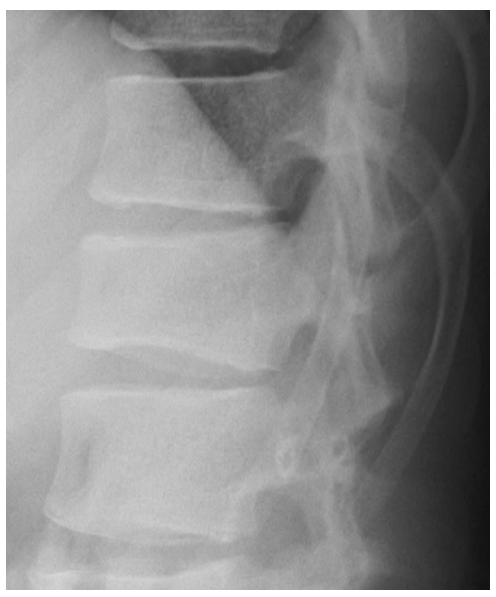

(b)

Figure 5. (a) Five months after the initiation of chemotherapy, he sustained a fall again, and radiographs showed L1 and $L 2$ compression fractures associated with steroid-induced osteoporosis; (b) Five years after disease onset, the lower heights of the $T 12$ and $L 2$ vertebral bodies improved as he grew, but $L 1$ showed little change. 
their retrospective study, Chin et al. reported that of the 39 consecutive patients, 11 had paraplegia and 14 had sphincter dysfunction [10].

MRI is a useful diagnostic imaging tool by which hematopoietic malignant tumors, including malignant lymphomas, can be visualized as areas showing uniform diffuse infiltration of the bone marrow [11,16-18]. Axial MRI sometimes shows the so-called "wrap-around" sign characteristic of small round cell tumors, including malignant lymphomas, reflecting infiltration in the area surrounding the tumor, with maintained continuity of the bone cortex [19]. This sign was observed in the MRI from our patient as well.

However, biopsy is usually needed because definitive diagnosis is difficult by using imaging alone. CT-guided biopsy, which we adopted in this case, is less invasive and safer; this technique also provides a higher diagnostic yield. Puri et al. have reported that the overall diagnostic yield and accuracy for bony lesions on CT-guided core-needle biopsies were $81.03 \%$ and $95.74 \%$, respectively, and those for soft-tissue lesions were $70 \%$ and $92.85 \%$, respectively [20].

Because hematogenous dissemination occurs early in the course of NHL in children, multiagent chemotherapy is considered as the first-line of therapy for pediatric malignant lymphoma [2]. We performed multiagent chemotherapy, including intrathecal injection without irradiation, according to a Japanese clinical trial based on previous trials $[13,21,22]$. According to the LMB89 study, intrathecal chemotherapy had a similar outcome to cranial irradiation in patients with CNS involvement [13]. Moreover, in 5-year survivors of pediatric NHL, chest radiation therapy was a risk factor for deaths from other causes (rate ratio, 1.9) and deaths from solid second malignant neoplasms (rate ratio, 7.5) [23]. In a study in 31 patients with NHL arising in bone, 5-year EFS in Murphy stages I and II was $94.1 \%$ and EFS in Murphy stages III was $70.7 \%$ [24]. Fortunately, our patient presenting with large B-cell lymphoma showed 5 years complete remission without neurological involvement, although tumor mass was found to advance into the spinal canal. In addition, according to the case of eosinophilic granuloma of the spine, treatment with immobilization in brace was sufficient to allow for vertebral remodeling [25]. Similarly, we thought significant reduction in the size of the tumor and immobilization in spinal orthosis before his growth spurt might have been effective for restoration of vertebral height.

\section{Conclusion}

Herein, we present a rare case of pediatric primary malignant lymphoma of the spine. The patient responded well to multiagent chemotherapy with 5 years of complete remission, and the deformed vertebral bodies im- proved without any neurological deficits.

\section{REFERENCES}

[1] R. A. Eeles, P. O’Brien, A. Horwich and M. Brada , "NonHodgkin's Lymphoma Presenting with Extradural Spinal Cord Compression: Functional Outcome and Survival," British Journal of Cancer, Vol. 63, No. 1, 1991, pp. 126129. doi:10.1038/bjc. 1991.25

[2] J. T. Sandlund, J. R. Downing and W. M. Crist, "NonHodgkin's Lymphoma in Childhood," The New England Journal of Medicine, Vol. 334, No. 19, 1996, pp. 12381248. doi:10.1056/NEJM199605093341906

[3] R. K. Fairbanks, J. A. Bonner, C. Y. Inwards, J. G. Strickler, T. M. Habermann, K. K. Unni and J. Su, "Treatment of Stage IE Primary Lymphoma of Bone," International Journal of Radiation Oncology, Biology, Physics, Vol. 28, No. 2, 1994, pp. 363-372.

doi:10.1016/0360-3016(94)90059-0

[4] C. Freeman, J. W. Berg and S. J. Cutler, "Occurrence and Prognosis of Extranodal Lymphomas," Cancer, Vol. 29, No. 1, 1972, pp. 252-260.

doi:10.1002/1097-0142(197201)29:1<252::AID-CNCR28 20290138>3.0.CO;2-\#

[5] E. Barbieri, S. Cammelli, F. Mauro, F. Perini, A. Cazzola, S. Neri, F. Bunkheila, S. Ferrari, V. Brandoli, P. Zinzani, M. Mercuri and G. Bacci, "Primary Non-Hodgkin's Lymphoma of the Bone: Treatment and Analysis of Prognostic Factors for Stage I and Stage II," International Journal of Radiation Oncology, Biology, Physics, Vol. 59, No. 3, 2004, pp. 760-764. doi:10.1016/j.ijrobp.2003.11.020

[6] A. J. Rathmell, M. K. Gospodarowicz, S. B. Sutcliffe and R. M. Clark, "Localised Lymphoma of Bone: Prognostic Factors and Treatment Recommendations. The Princess Margaret Hospital Lymphoma Group," British Journal of Cancer, Vol. 66, No. 3, 1992, pp. 603-606.

[7] P. L. Zinzani, G. Carrillo, S. Ascani, E. Barbieri, M. Tani, M. Paulli, V. Stefoni, E. Sabattini, L. Alinari, R. Binazzi, S. Tura, M. Baccarani and S. A. Pileri, "Primary Bone Lymphoma: Experience with 52 Patients," Haematologica, Vol. 88, No. 3, 2003, pp. 280-285.

[8] M. P. Glotzbecker, L. S. Kersun, J. K. Choi, B. P. Wills, A. A. Schaffer and J. P. Dormans, "Primary Non-Hodgkin's Lymphoma of Bone in Children," The Journal of Bone and Joint Surgery. American Volume, Vol. 88, No. 3, 2006, pp. 583-594. doi:10.2106/JBJS.D.01967

[9] D. Limb, C. Dreghorn, J. K. Murphy and R. Mannion, "Primary Lymphoma of Bone," International Orthopaedics, Vol. 18, No. 3, 1994, pp. 180-183. doi:10.1007/BF00192476

[10] H. Y. Ching, J. M. Horsman, C. R. Radstone, H. Hancock, W. R. Timperley and B. W. Hancock, "Non-Hodgkin's Lymphoma Presenting with Spinal Involvement: The Sheffield Lymphoma Group Experience (1970-2000)," International Journal of Oncology, Vol. 19, No. 1, 2001, pp. 149-156.

[11] L. A. Moulopoulos and M. A. Dimopoulos, "Magnetic Resonance Imaging of the Bone Marrow in Hematologic Malignancies," Blood, Vol. 90, No. 6, 1997, pp. $2127-$ 
2147.

[12] S. B. Murphy, "Childhood Non-Hodgkin's Lymphoma," The New England Journal of Medicine, Vol. 299, No. 26, 1978, pp. 1446-1448.

doi:10.1056/NEJM197812282992606

[13] C. Patte, A. Auperin, J. Michon, H. Behrendt, G. Leverger, D. Frappaz, P. Lutz, C. Coze, Y. Perel, M. Raphaël and M. J. Terrier-Lacombe, "The Société Française D'Oncologie Pédiatrique LMB89 Protocol: Highly Effective Multiagent Chemotherapy Tailored to the Tumor Burden and Initial Response in 561 Unselected Children with B-Cell Lymphomas and L3 Leukemia," Blood, Vol. 97, No. 11, 2001, pp. 3370-3379. doi:10.1182/blood.V97.11.3370

[14] M. Nakano, N. Hirano, H. Ishihara, Y. Kawaguchi, H. Watanabe and K. Matsuura, "Calcium Phosphate CementBased Vertebroplasty Compared with Conservative Treatment for Osteoporotic Compression Fractures: A Matched Case-Control Study," Journal of Neurosurgery: Spine, Vol. 4. No. 2, 2006, pp. 110-117. doi:10.3171/spi.2006.4.2.110

[15] J. J. Vaughan, R. B. Winter, J. E. Lonstein, J. R. Johnson and J. E. Dunnington, "Non-Hodgkin Lymphoma of the Spine: A Report of Three Cases with a Minimum TenYear Follow-Up," The Journal of Bone and Joint Surgery, Vol. 80. No. 1, 1998, pp. 104-110.

[16] H. J. Kim, K. N. Ryu, W. S. Choi, B. K. Choi, J. M. Choi and Y. Yoon, "Spinal Involvement of Hematopoietic Malignancies and Metastasis: Differentiation Using MR Imaging," Clinical Imaging, Vol. 23, No. 2, 1999, pp. 125133. doi:10.1016/S0899-7071(99)00105-9

[17] M. D. Häussler, M. J. Fenstermacher, D. A. Johnston and T. S. Harle, "MRI of Primary Lymphoma of Bone: Cortical Disorder as a Criterion for Differential Diagnosis," Journal of Magnetic Resonance Imaging, Vol. 9, No. 1, 1999, pp. 93-100. doi:10.1002/(SICI)1522-2586(199901)9:1<93::AID-JMR I13>3.0.CO;2-D

[18] A. Linden, R. Zankovich, P. Theissen, V. Diehl and H. Schicha, "Malignant Lymphoma: Bone Marrow Imaging versus Biopsy," Radiology, Vol. 173, No. 2, 1989, pp. 335-339.

[19] L. A. Moulopoulos, M. A. Dimopoulos, A. Vourtsi, A. Gouliamos and L. Vlahos, "Bone Lesions with Soft-Tis- sue Mass: Magnetic Resonance Imaging Diagnosis of Lymphomatous Involvement of the Bone Marrow versus Multiple Myeloma and Bone Metastases," Leukemia \& Lymphoma, Vol. 34, No. 1-2, 1999, pp. 179-184.

[20] A. Puri, V. U. Shingade, M. G. Agarwal, C. Anchan, S. Juvekar, S. Desai and N. A. Jambhekar, "CT-Guided Percutaneous Core Needle Biopsy in Deep Seated Musculoskeletal Lesions: A Prospective Study of 128 Cases," Skeletal Radiology, Vol. 35, No. 3, 2006, pp. 138-143. doi:10.1007/s00256-005-0038-4

[21] A. Reiter, M. Schrappe, M. Tiemann, W. D. Ludwig, E. Yakisan, M. Zimmermann, G. Mann, A. Chott, W. Ebell, T. Klingebiel, N. Graf, B. Kremens, S. Müller-Weihrich, H. J. Plüss, F. Zintl, G. Henze and H. Riehm, "Improved Treatment Results in Childhood B-Cell Neoplasms with Tailored Intensification of Therapy: A Report of the Berlin-Frankfurt-Münster Group Trial NHL-BFM 90," Blood, Vol. 94, No. 10, 1999, pp. 3294-3306.

[22] M. Tsurusawa, T. Taga, Y. Horikoshi, A. Ogawa, A. Kikuta, H. Kanegane, T. Matsushita, N. Hyakuna, Y. Shimomura and K. Ohshima, "Favourable Outcomes in Children with Diffuse Large B-Cell Lymphoma Treated by a Short-Term ALL-Like Regimen: A Report on the NHL 960 Study from the Japanese Childhood Cancer and Leukemia Study Group," Leukemia \& Lymphoma, Vol. 49, No. 4, 2008, pp. 734-739. doi:10.1080/10428190701851356

[23] E. C. Bluhm, C. Ronckers, R. J. Hayashi, J. P. Neglia, A. C. Mertens, M. Stovall, A. T. Meadows, P. A. Mitby, J. A. Whitton, S. Hammond, J. D. Barker, S. S. Donaldson, L. L. Robison and P. D. Inskip, "Cause-Specific Mortality and Second Cancer Incidence after Non-Hodgkin Lymphoma: A Report from the Childhood Cancer Survivor Study," Blood, Vol. 111, No. 8, 2008, pp. 4014-4021. doi:10.1182/blood-2007-08-106021

[24] M. A. Lones, S. L. Perkins, R. Sposto, N. Tedeschi, M. E. Kadin, C. R. Kjeldsberg, J. F. Wilson, D. L. Zwick and M. S. Cairo, "Non-Hodgkin's Lymphoma Arising in Bone in Children and Adolescents Is Associated with an Excellent Outcome: A Children's Cancer Group Report," Journal of Clinical Oncology, Vol. 20, No. 9, 2002, pp. 2293-2301.

[25] P. Raab, F. Hohmann, J. Kühl and R. Krauspe, "Vertebral Remodeling in Eosinophilic Granuloma of the Spine. A Long-Term Follow-Up," Spine, Vol. 23, No. 12, 1998, pp. 1351-1354. doi:10.1097/00007632-199806150-00011 